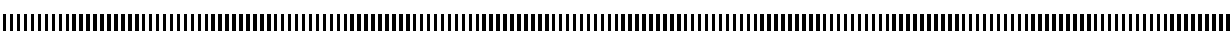

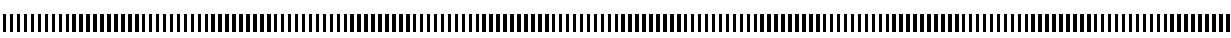
|

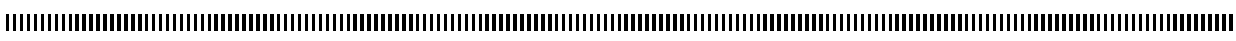
|

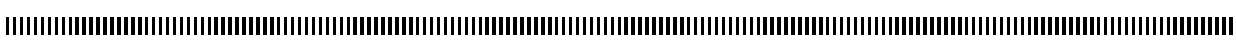

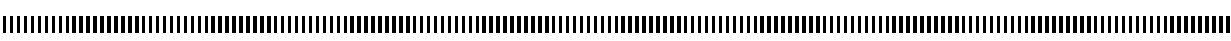

CARI'10

\title{
Sur un modèle de compétition et de coexistence dans le chémostat
}

\section{Radhouane Fekih Salem a,b,* — Tewfik Sari a,c _ Nahla Abdellatif b,d}

a EPI Modemic INRA-INRIA, UMR Mistea, SupAgro Bât 21,

2, place Pierre Viala, 34060 Montpellier, France.

b Université de Tunis El Manar, Ecole Nationale d'Ingénieurs de Tunis,

Laboratoire de Modélisation Mathématique et Numérique dans les Sciences de l'Ingénieur, B.P. 37, 1002 Tunis Le Belvédère, Tunisie.

fekih@supagro.inra.fr

c Cemagref, UMR Itap,

361 rue Jean-François Breton, 34196 Montpellier Cedex, France.

tewfik.sari@cemagref.fr

d Ecole Nationale des Sciences de l'Informatique,

Campus Universitaire de Manouba, 2010, Manouba, Tunisie.

nahla.abdellatif@ensi.rnu.tn

* Corresponding author.

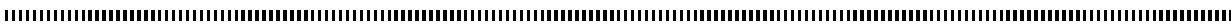

RÉSUMÉ. Dans ce travail, nous considérons le modèle mathématique de deux espèces microbiennes en compétition sur une seule ressource dans un chémostat, où on prend en considération les interactions interspécifiques entre les deux populations de micro-organismes et les interactions intraspécifiques entre les individus eux-mêmes. Les fonctions de croissance sont strictement monotones et les taux de dilution sont distincts. Nous déterminons les points d'équilibre, ainsi que leur stabilité locale.

ABSTRACT. In this paper, we consider the mathematical model of two microbial species competition on a single resource in a chemostat. We take into account the interspecific interactions between the two populations of micro-organisms and intraspecific interactions between individuals themselves. The growth functions are monotonic and the dilution ratios are distinct. We determine the equilibrium points, and their local stability.

MOTS-CLÉS : Compétition, intraspécifique, interspécifique, coexistence d'espèces, chémostat

KEYWORDS : Competition, intraspecific, interspecific, Species coexistence , chemostat

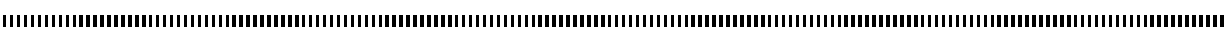

ARIMA, Vol. 14 - pp. 15-30 


\section{Introduction}

Le chémostat est un appareil de laboratoire qui permet la culture et l'étude d'espèces de micro-organismes. Dans le modèle mathématique de la compétition pour une ressource dans un chémostat, un résultat classique, connu sous le nom de Principe d'Exclusion Compétitive, affirme qu'une seule espèce peut survivre à la compétition $[11,12,13]$. Le modèle s'écrit

$$
\begin{aligned}
\dot{S} & =D\left(S^{0}-S\right)-\sum_{i=1}^{n} \frac{1}{y_{i}} f_{i}(S) X_{i}, \\
\dot{X}_{i} & =X_{i}\left(f_{i}(S)-D\right), \quad i=1 \cdots n .
\end{aligned}
$$

où $S(t)$ désigne la concentration du substrat à l'instant $t$ (nutriment), $X_{i}(t)$ désigne la concentration de l'espèce $i$ (biomasse) avec $n$ le nombre d'espèces. $S^{0}$ est la concentration du substrat à l'entrée du chémostat et $D$ est le taux de dilution dans le chémostat. La fonction $f_{i}(S)$ représente le taux de croissance de l'espèce $i$ qu'on suppose strictement croissante et $y_{i}$ est un coefficient de rendement.

Si on suppose que $\lambda_{1}<\lambda_{j}<S^{0}$, pour $j \geqslant 2$ où $\lambda_{i}:=f_{i}^{-1}(D)$, alors toutes les solutions tendent vers l'équilibre globalement asymptotiquement stable

$$
S=\lambda_{1}, \quad X_{1}=y_{1}\left(S^{0}-\lambda_{1}\right), \quad X_{j}=0, j \geqslant 2 .
$$

Ce résultat mathématique contredit la grande biodiversité que l'on trouve dans les milieux naturels ainsi que dans les bioréacteurs. Pour construire des modèles mathématiques qui soient plus en accord avec les observations, plusieurs améliorations du modèle idéalisé de la compétition ont été proposées $[1,2,3,5,6,7,8,9,10,13,14]$. On se propose d'étudier ici le modèle suivant

$$
\begin{aligned}
& \dot{S}=D\left(S^{0}-S\right)-\sum_{i=1}^{2} \frac{1}{y_{i}} f_{i}(S) X_{i}, \\
& \dot{X}_{i}=X_{i}\left(f_{i}(S)-\sum_{j=1}^{2} q_{i j}\left(X_{j}\right)-D_{i}\right), \quad i=1,2
\end{aligned}
$$

où l'on prend en considération des termes de compétition intraspécifique $q_{i i}\left(X_{i}\right)$ ainsi que des termes de compétition interspécifique supplémentaires $q_{i j}\left(X_{j}\right)$ entre les espèces.

Ce modèle a été proposé et étudié dans [14], dans le cas de la compétition interspécifique $\left(q_{11}=q_{22}=0\right)$, et dans le cas de la compétition intraspécifique $\left(q_{12}=q_{21}=0\right)$. Le cas, où l'on considère une compétition interspécifique dans la dynamique de l'espèce $X_{1}$ $\left(q_{11}=0\right)$ et une compétition intraspécifique dans la dynamique de l'espèce $X_{2}\left(q_{21}=0\right)$ n'a pas été considéré dans [14]. Ce cas semble aussi pertinent pour les applications car il présente une certaine analogie avec le modèle de coexistence de deux espèces grâce à la production d'un métabolite $[3,4]$ :

$$
\left\{\begin{array}{l}
\dot{S}=D\left(S^{0}-S\right)-f_{1}(S) X_{1}-f_{2}(S) X_{2} \\
\dot{X}_{1}=X_{1}\left(f_{1}(S)+g_{1}(R)-d_{1} R-D\right) \\
\dot{X}_{2}=X_{2}\left(f_{2}(S)-d_{2} R-D\right) \\
\dot{R}=f_{2}(S) X_{2}-b g_{1}(R) X_{1}-D R
\end{array}\right.
$$


Dans ce modèle, l'espèce $X_{2}$ produit un métabolite $R$ qui inhibe sa propre croissance et vient activer et inhiber à la fois la croissance de l'espèce $X_{1}$. Le but de cet article est de comprendre le mécanisme de coexistence dans ce type modèle et de voir par exemple si dans le modèle simplifié du type suivant

$$
\left\{\begin{array}{l}
\dot{S}=D\left(S^{0}-S\right)-\frac{1}{y_{1}} f_{1}(S) X_{1}-\frac{1}{y_{2}} f_{2}(S) X_{2} \\
\dot{X}_{1}=X_{1}\left(f_{1}(S)-q_{1}\left(X_{2}\right)-D_{1}\right) \\
\dot{X}_{2}=X_{2}\left(f_{2}(S)-q_{2}\left(X_{2}\right)-D_{2}\right) .
\end{array}\right.
$$

où l'espèce $X_{2}$ inhibe sa propre croissance, ainsi que celle de $X_{1}$, il peut y avoir coexistence des espèces. On suppose que

- $f_{i}(0)=0$ et pour tout $S>0, f_{i}^{\prime}(S)>0$, avec $i=1,2$.

$-q_{i}(0)=0$ et pour tout $X>0, q_{i}^{\prime}(X)>0$.

On montre le résultat suivant :

\section{Proposition 1.1}

- Pour des valeurs initiales positives, les solutions de (1) restent positives au cours $d u$ temps et sont bornées quand $t \rightarrow+\infty$.

- L'ensemble $\Omega=\left\{\left(S, X_{1}, X_{2}\right) \in \mathbb{R}_{+}^{3}: Z=S+\frac{X_{1}}{y_{1}}+\frac{X_{2}}{y_{2}} \leqslant \max \left(Z(0), \frac{D}{D^{*}} S^{0}\right)\right\}$ est attracteur positif invariant de toutes les solutions du système (1), avec

$$
D^{*}=\min \left(D, D_{1}, D_{2}\right) \text {. }
$$

Preuve. Dès que $S(t)$ s'annule, on a $\dot{S}(t)=D S^{0}>0$, alors $S(t) \geqslant 0$, pour tout $t$. Pour toute condition initiale $X_{i}\left(t_{0}\right)$ positive, dès qu'il existe un premier temps $t_{1}$ tel que $X_{i}\left(t_{1}\right)=0$, on a $\dot{X}_{i}\left(t_{1}\right)=0$, c'est à dire $X_{i}(t)$ reste identiquement nulle, ce qui prouve la positivité. Pour montrer que toutes les solutions sont bornées, on a

$$
\dot{Z}=D\left(S^{0}-S\right)-D_{1} \frac{X_{1}}{y_{1}}-D_{2} \frac{X_{2}}{y_{2}}-q_{1}\left(X_{2}\right) \frac{X_{1}}{y_{1}}-q_{2}\left(X_{2}\right) \frac{X_{2}}{y_{2}} .
$$

Par majoration, on obtient

$$
\dot{Z} \leqslant D S^{0}-D^{*} Z=D^{*}\left[\frac{D}{D^{*}} S^{0}-Z\right] .
$$

On pose $V=Z-\frac{D}{D^{*}} S^{0}$, alors on a $\dot{V} \leqslant-D^{*} V$. Par suite,

$$
Z(t) \leqslant \frac{D}{D^{*}} S^{0}+\left(Z(0)-\frac{D}{D^{*}} S^{0}\right) e^{-D^{*} t} \leqslant \max \left(Z(0), \frac{D}{D^{*}} S^{0}\right) .
$$

Alors, pour toutes solutions initiales dans $\Omega$, les solutions sont bornées et par suite l'ensemble $\Omega$ est attracteur invariant avec

$$
\lim _{t \rightarrow+\infty} Z(t) \leqslant \frac{D}{D^{*}} S^{0}
$$




\section{Modèle de Compétition interspécifique}

On se propose dans un premier temps d'étudier le modèle de compétition interspécifique entre $X_{1}$ et $X_{2}$ avec des termes de compétition intraspécifique nuls $\left(q_{2}=0\right)$. Le modèle s'écrit

$$
\left\{\begin{array}{l}
\dot{S}=D\left(S^{0}-S\right)-\frac{1}{y_{1}} f_{1}(S) X_{1}-\frac{1}{y_{2}} f_{2}(S) X_{2} \\
\dot{X}_{1}=X_{1}\left(f_{1}(S)-q_{1}\left(X_{2}\right)-D_{1}\right) \\
\dot{X}_{2}=X_{2}\left(f_{2}(S)-D_{2}\right)
\end{array}\right.
$$

\subsection{Existence des équilibres}

On étudie l'existence des points d'équilibre non-négatifs du système (2), on calcule les isoclines nulles, on obtient

$$
\left\{\begin{array}{l}
D\left(S^{0}-S\right)=\frac{1}{y_{1}} f_{1}(S) X_{1}+\frac{1}{y_{2}} f_{2}(S) X_{2} \\
X_{1}=0 \quad \text { ou } \quad f_{1}(S)-D_{1}=q_{1}\left(X_{2}\right) \\
X_{2}=0 \quad \text { ou } \quad S=\lambda_{2}
\end{array}\right.
$$

où $\lambda_{i}:=f_{i}^{-1}\left(D_{i}\right)$, pour $i=1,2$. Comme la fonction $q_{1}($.$) est strictement croissante,$ alors on définit les fonctions

$$
\xi_{1}(S)=q_{1}^{-1}\left(f_{1}(S)-D_{1}\right) \quad \text { et } \quad \xi_{3}(S)=\frac{D y_{2}\left(S^{0}-S\right)}{f_{2}(S)} .
$$

Proposition 2.1 Les points d'équilibre du système (2) sont données par

- $E_{0}=\left(S^{0}, 0,0\right)$ qui existe toujours et représente le lessivage.

- $E_{1}=\left(\lambda_{1}, \hat{X}_{1}, 0\right)$ qui existe si et seulement si $S^{0}>\lambda_{1}$, avec

$$
\hat{X}_{1}=\frac{D y_{1}\left(S^{0}-\lambda_{1}\right)}{D_{1}} .
$$

- $E_{2}=\left(\lambda_{2}, 0, \hat{X}_{2}\right)$ qui existe si et seulement si $S^{0}>\lambda_{2}$, avec

$$
\hat{X}_{2}=\frac{D y_{2}\left(S^{0}-\lambda_{2}\right)}{D_{2}}=\xi_{3}\left(\lambda_{2}\right) .
$$

$-\bar{E}=\left(\lambda_{2}, \bar{X}_{1}, \bar{X}_{2}\right)$ qui existe si et seulement si $\lambda_{1}<\lambda_{2}<S^{0}$ et $\xi_{3}\left(\lambda_{2}\right)>\xi_{1}\left(\lambda_{2}\right)$, avec

$$
\bar{X}_{2}=\xi_{1}\left(\lambda_{2}\right) \quad \text { et } \quad \bar{X}_{1}=\frac{y_{1}}{f_{1}\left(\lambda_{2}\right)} \frac{D_{2}}{y_{2}}\left[\xi_{3}\left(\lambda_{2}\right)-\xi_{1}\left(\lambda_{2}\right)\right] .
$$

Preuve. On peut facilement vérifier l'existence des points d'équilibre $E_{0}, E_{1}$ et $E_{2}$. Le point d'équilibre positif $\bar{E}=\left(\bar{S}, \bar{X}_{1}, \bar{X}_{2}\right)$ avec $\bar{S}>0$ et $\bar{X}_{i}>0$, pour $i=1,2$, représente la coexistence entre les deux espèces. De la deuxième et la troisième équation du système (3), on déduit (voir Fig. 1)

$$
\bar{S}=\lambda_{2} \quad \text { et } \quad \bar{X}_{2}=q_{1}^{-1}\left(f_{1}\left(\lambda_{2}\right)-D_{1}\right)=\xi_{1}\left(\lambda_{2}\right)
$$




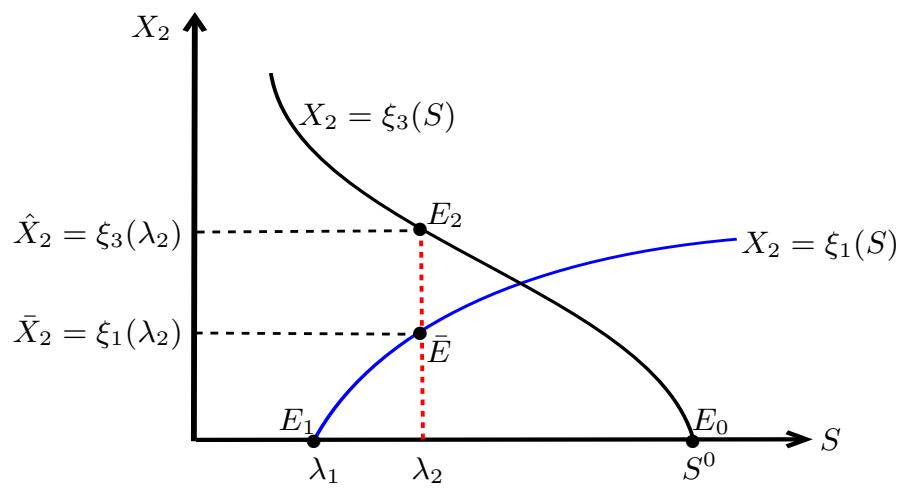

Figure 1. Projection des points d'équilibre $E_{0}, E_{1}, E_{2}$ et $\bar{E}$ dans le plan $\left(S, X_{2}\right)$ et Condition d'existence du point d'équilibre positif $\bar{E}$.

d'où $\bar{X}_{2}>0$ si et seulement si $\lambda_{1}<\lambda_{2}$. De la première équation du système (3),

$$
\bar{X}_{1}=\frac{y_{1}}{f_{1}\left(\lambda_{2}\right)}\left[D\left(S^{0}-\lambda_{2}\right)-\frac{1}{y_{2}} f_{2}\left(\lambda_{2}\right) \bar{X}_{2}\right]=\frac{y_{1}}{f_{1}\left(\lambda_{2}\right)} \frac{D_{2}}{y_{2}}\left[\xi_{3}\left(\lambda_{2}\right)-\bar{X}_{2}\right]
$$

donc $\bar{X}_{1}>0$ si et seulement si $\xi_{3}\left(\lambda_{2}\right)>\bar{X}_{2}$ et $\xi_{3}\left(\lambda_{2}\right)>0$, c'est à dire $\xi_{3}\left(\lambda_{2}\right)>\bar{X}_{2}$ et $\lambda_{2}<S^{0}$, ce qui achève la preuve de la proposition.

\subsection{Stabilité locale des points d'équilibre}

Dans la suite, on se propose d'étudier la stabilité locale des points d'équilibre du système (2).

Proposition 2.2 On suppose $\lambda_{1}<\lambda_{2}<S^{0}$.

- Si $\xi_{1}\left(\lambda_{2}\right)<\xi_{3}\left(\lambda_{2}\right)$ alors $\bar{E}$ est instable, $E_{1}$ et $E_{2}$ sont localement asymptotiquement stables (LAS), c'est à dire on a bistabilité avec deux bassins d'attraction.

- Si $\xi_{3}\left(\lambda_{2}\right)<\xi_{1}\left(\lambda_{2}\right)$ alors $\bar{E}$ n'existe pas, $E_{2}$ est instable et $E_{1}$ est LAS.

Preuve. On sait que $E_{1}=\left(\lambda_{1}, \hat{X}_{1}, 0\right)$ existe si et seulement si $\lambda_{1}<S^{0}$. La matrice Jacobienne en $E_{1}$ du système (2) est

$$
\mathbf{J}_{\mathbf{1}}=\left(\begin{array}{ccc}
-D-\frac{f_{1}^{\prime}\left(\lambda_{1}\right)}{y_{1}} \hat{X}_{1} & -\frac{D_{1}}{y_{1}} & -\frac{f_{2}\left(\lambda_{1}\right)}{y_{2}} \\
f_{1}^{\prime}\left(\lambda_{1}\right) \hat{X}_{1} & 0 & -q_{1}^{\prime}(0) \hat{X}_{1} \\
0 & 0 & f_{2}\left(\lambda_{1}\right)-D_{2}
\end{array}\right)
$$

Donc $f_{2}\left(\lambda_{1}\right)-D_{2}$ est une valeur propre de $\mathbf{J}_{\mathbf{1}}$. Cette valeur propre est négative si et seulement si $\lambda_{1}<\lambda_{2}$. Les deux autres valeurs propres de $\mathbf{J}_{\mathbf{1}}$ sont les valeurs propres de la matrice

$$
A=\left(\begin{array}{cc}
-D-\frac{f^{\prime}\left(\lambda_{1}\right)}{y_{1}} \hat{X}_{1} & -\frac{D_{1}}{y_{1}} \\
f_{1}^{\prime}\left(\lambda_{1}\right) \hat{X}_{1} & 0
\end{array}\right)
$$

On a

$$
\operatorname{det} A=\frac{D_{1}}{y_{1}} f_{1}^{\prime}\left(\lambda_{1}\right) \hat{X}_{1}>0, \quad \operatorname{tr} A=-D-\frac{f^{\prime}\left(\lambda_{1}\right)}{y_{1}} \hat{X}_{1}<0 .
$$


Par conséquent, les deux valeurs propres de $A$ sont à partie réelle strictement négative. On en déduit que $E_{1}$ est LAS si et seulement si $\lambda_{1}<S^{0}$ et $\lambda_{1}<\lambda_{2}$.

La matrice Jacobienne en $E_{2}$ du système (2) est égale à

$$
\mathbf{J}_{\mathbf{2}}=\left(\begin{array}{ccc}
-D-\frac{f_{2}^{\prime}\left(\lambda_{2}\right)}{y_{2}} \hat{X}_{2} & -\frac{f_{1}\left(\lambda_{2}\right)}{y_{1}} & -\frac{D_{2}}{y_{2}} \\
0 & f_{1}\left(\lambda_{2}\right)-q_{1}\left(\hat{X}_{2}\right)-D_{1} & 0 \\
f_{2}^{\prime}\left(\lambda_{2}\right) \hat{X}_{2} & 0 & 0
\end{array}\right)
$$

Donc $f_{1}\left(\lambda_{2}\right)-q_{1}\left(\hat{X}_{2}\right)-D_{1}$ est une valeur propre de $\mathbf{J}_{\mathbf{2}}$. Cette valeur propre est négative si et seulement si $f_{1}\left(\lambda_{2}\right)-D_{1}<q_{1}\left(\hat{X}_{2}\right)$, c'est à dire $\xi_{1}\left(\lambda_{2}\right)<\hat{X}_{2}=\xi_{3}\left(\lambda_{2}\right)$. Les deux autres valeurs propres de $\mathbf{J}_{2}$ sont les valeurs propres de la matrice

$$
A=\left(\begin{array}{cc}
-D-\frac{f_{2}^{\prime}\left(\lambda_{2}\right)}{y_{2}} \hat{X}_{2} & -\frac{D_{2}}{y_{2}} \\
f_{2}^{\prime}\left(\lambda_{2}\right) \hat{X}_{2} & 0
\end{array}\right)
$$

On a

$$
\operatorname{det} A=\frac{D_{2}}{y_{2}} f_{2}^{\prime}\left(\lambda_{2}\right) \hat{X}_{2}>0, \quad \operatorname{tr} A=-D-\frac{f^{\prime}\left(\lambda_{2}\right)}{y_{2}} \hat{X}_{2}<0 .
$$

Par conséquent, les deux valeurs propres de $A$ sont à partie réelle strictement négative. $E_{2}$ est donc LAS si et seulement si $\lambda_{2}<S^{0}$ et $\xi_{1}\left(\lambda_{2}\right)<\xi_{3}\left(\lambda_{2}\right)$.

La matrice Jacobienne en $\bar{E}$ du système (2) est égale à

$$
\mathbf{J}_{\overline{\mathbf{E}}}=\left(\begin{array}{ccc}
-m_{11} & -m_{12} & -m_{13} \\
m_{21} & 0 & -m_{23} \\
m_{31} & 0 & 0
\end{array}\right)
$$

avec

$$
\begin{gathered}
m_{11}=D+\frac{f_{1}^{\prime}\left(\lambda_{2}\right)}{y_{1}} \bar{X}_{1}+\frac{f_{2}^{\prime}\left(\lambda_{2}\right)}{y_{2}} \bar{X}_{2}, \quad m_{12}=\frac{f_{1}\left(\lambda_{2}\right)}{y_{1}}, \quad m_{13}=\frac{D_{2}}{y_{2}} \\
m_{21}=f_{1}^{\prime}\left(\lambda_{2}\right) \bar{X}_{1}, \quad m_{23}=q_{1}^{\prime}\left(\bar{X}_{2}\right) \bar{X}_{1}, \quad m_{31}=f_{2}^{\prime}\left(\lambda_{2}\right) \bar{X}_{2} .
\end{gathered}
$$

Le polynôme caractéristique est donné par

$$
P_{J}(\lambda)=a_{0} \lambda^{3}+a_{1} \lambda^{2}+a_{2} \lambda+a_{3}
$$

avec

$$
a_{0}=-1, \quad a_{1}=-m_{11}, \quad a_{2}=-\left(m_{12} m_{21}+m_{13} m_{31}\right), \quad a_{3}=m_{12} m_{31} m_{23} .
$$

Comme les coefficients $a_{i}$ ne gardent pas un signe constant, alors d'après le critère de Routh-Hurwitz, $\bar{E}$ est instable.

L'ajout du terme de compétition interspécifique $q_{1}\left(X_{2}\right)$ pour inhiber la croissance de $X_{1}$ par $X_{2}$, a pu faire apparaître un équilibre positif de coexistence $\bar{E}$ instable qui sépare deux bassins d'attraction où $E_{1}$ et $E_{2}$ sont localement asymptotiquement stables. Dans la suite, on se propose d'ajouter un terme de compétition intraspécifique $q_{2}\left(X_{2}\right)$ entre les individus de la deuxième espèce, pour espérer avoir la coexistence entre les deux espèces et pour avoir un modèle plus approprié avec les observations. 


\section{Modèle de Compétition interspécifique et intraspécifique}

\subsection{Existence des équilibres}

On se propose d'étudier l'existence des points d'équilibre du système (1). On note

$$
\xi_{2}(S)=q_{2}^{-1}\left(f_{2}(S)-D_{2}\right)
$$

Proposition 3.1 Les points d'équilibre du système (1) sont données par

- $E_{0}=\left(S^{0}, 0,0\right)$ qui existe toujours.

- $E_{1}=\left(\lambda_{1}, \hat{X}_{1}, 0\right)$ qui existe et est unique si $\lambda_{1}<S^{0}$ avec

$$
\hat{X}_{1}=\frac{D y_{1}\left(S^{0}-\lambda_{1}\right)}{D_{1}} .
$$

$-E_{2}=\left(\hat{S}, 0, \hat{X}_{2}\right)$ qui existe et est unique si et seulement si $\lambda_{2}<S^{0}$, avec $\hat{S}$ est solution de l'équation $\xi_{2}(S)=\xi_{3}(S)$ et

$$
\hat{X}_{2}=\xi_{2}(\hat{S})=\xi_{3}(\hat{S}) .
$$

$-\bar{E}=\left(\bar{S}, \bar{X}_{1}, \bar{X}_{2}\right)$ qui existe si $\lambda_{1}<\lambda_{2}<S^{0}, \xi_{2}\left(S^{0}\right)>\xi_{1}\left(S^{0}\right)$ et $\xi_{3}(\bar{S})>\xi_{2}(\bar{S})$, avec $\bar{S}$ est solution de l'équation $\xi_{2}(S)=\xi_{1}(S)$,

$$
\bar{X}_{2}=\xi_{1}(\bar{S})=\xi_{2}(\bar{S}) \quad \text { et } \quad \bar{X}_{1}=\frac{y_{1}}{f_{1}(\bar{S})} \frac{f_{2}(\bar{S})}{y_{2}}\left(\xi_{3}(\bar{S})-\bar{X}_{2}\right) .
$$

Preuve. On peut facilement vérifier l'existence des points d'équilibre $E_{0}$ et $E_{1}$. Le troisième point est donné par $E_{2}=\left(\hat{S}, 0, \hat{X}_{2}\right)$ avec $\hat{S} \neq 0, \hat{X}_{2} \neq 0$, et tels que

$$
\left\{\begin{array}{l}
\hat{X}_{2}=\frac{D y_{2}\left(S^{0}-\hat{S}\right)}{f_{2}(\hat{S})}=\xi_{3}(\hat{S}) \\
\hat{X}_{2}=q_{2}^{-1}\left(f_{2}(\hat{S})-D_{2}\right)=\xi_{2}(\hat{S}) .
\end{array}\right.
$$

On considère la fonction $\xi(S)=\xi_{2}(S)-\xi_{3}(S)$ de classe $C^{1}$ avec $\xi^{\prime}(S)>0$. Si $S^{0}<\lambda_{2}$, alors on a

$$
\xi\left(S^{0}\right)=\xi_{2}\left(S^{0}\right)<0 \quad \text { et } \quad \xi\left(\lambda_{2}\right)=-\xi_{3}\left(\lambda_{2}\right)>0 .
$$

Par suite, d'après le théorème des valeurs intermédiaires, il existe une solution

$$
\hat{S} \in] S^{0}, \lambda_{2}\left[\quad \text { telle que } \xi_{2}(\hat{S})=\xi_{3}(\hat{S})=\hat{X}_{2}<0,\right.
$$

c'est à dire $E_{2}$ n'est pas un point d'équilibre positif. Si $\lambda_{2}<S^{0}$, alors on a

$$
\xi\left(\lambda_{2}\right)=-\xi_{3}\left(\lambda_{2}\right)<0 \quad \text { et } \quad \xi\left(S^{0}\right)=\xi_{2}\left(S^{0}\right)>0,
$$

l'application du théorème des valeurs intermédiaires, permet de montrer qu'il existe

$$
\hat{S} \in] \lambda_{2}, S^{0}\left[\text { telle que } \xi_{3}(\hat{S})=\xi_{2}(\hat{S})=\hat{X}_{2}>0 .\right.
$$

Vol. 14 - pp. $15-30$ 


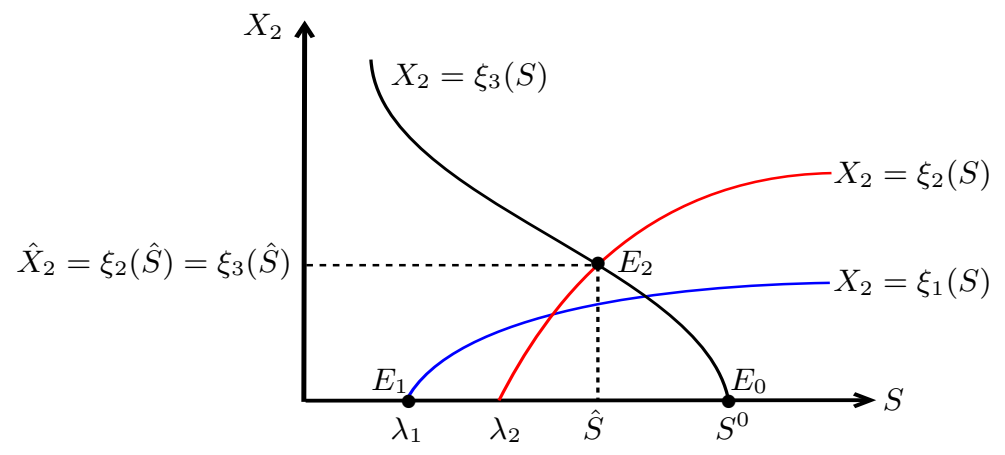

Figure 2. Condition d'existence du point d'équilibre $E_{2}$.

Comme $\xi(S)$ est strictement croissante sur $\left[\lambda_{2}, S^{0}\right]$, on conclut donc que $E_{2}$ existe et est unique si et seulement si $\lambda_{2}<S^{0}$ (voir Fig. 2).

Le dernier point d'équilibre est donné par $\bar{E}=\left(\bar{S}, \bar{X}_{1}, \bar{X}_{2}\right)$ avec $\bar{S}>0$ et $\bar{X}_{2}>0$ sont les solutions du système :

$$
\left\{\begin{array}{l}
\bar{X}_{2}=q_{1}^{-1}\left(f_{1}(\bar{S})-D_{1}\right)=\xi_{1}(\bar{S}) \\
\bar{X}_{2}=q_{2}^{-1}\left(f_{2}(\bar{S})-D_{2}\right)=\xi_{2}(\bar{S})
\end{array}\right.
$$

et $\bar{X}_{1}>0$ donné par

$$
D\left(S^{0}-\bar{S}\right)=\frac{1}{y_{1}} f_{1}(\bar{S}) \bar{X}_{1}+\frac{1}{y_{2}} f_{2}(\bar{S}) \bar{X}_{2}
$$

Pour avoir $\bar{X}_{2}>0$, il faut que $\lambda_{i}<\bar{S}$, pour $i=1,2$. De plus, pour que $\bar{X}_{1}>0$, il faut que $\bar{S}<S^{0}$. Une condition suffisante mais non nécessaire d'existence de $\bar{E}$ est que $\lambda_{1}<\lambda_{2}<S^{0}, \xi_{1}\left(S^{0}\right)<\xi_{2}\left(S^{0}\right)$ et de plus $\bar{X}_{1}>0$. En effet, dans ce cas, on considère la fonction $\xi(S)=\xi_{2}(S)-\xi_{1}(S)$ continue $\operatorname{sur}\left[\lambda_{2}, S^{0}\right]$ où

$$
\xi\left(\lambda_{2}\right)<0 \quad \text { et } \quad \xi\left(S^{0}\right)>0
$$

alors d'après le théorème des valeurs intermédiaires, il existe au moins $\bar{S} \in] \lambda_{2}, S^{0}$ [ tel que $\xi_{1}(\bar{S})=\xi_{2}(\bar{S})=\bar{X}_{2}$. Notons que l'on a

$$
\bar{X}_{1}=\frac{y_{1}}{f_{1}(\bar{S})}\left[D\left(S^{0}-\bar{S}\right)-\frac{1}{y_{2}} f_{2}(\bar{S}) \bar{X}_{2}\right]=\frac{y_{1}}{f_{1}(\bar{S})} \frac{f_{2}(\bar{S})}{y_{2}}\left[\xi_{3}(\bar{S})-\bar{X}_{2}\right] .
$$

D'où $\bar{X}_{1}>0$ si et seulement si $\bar{X}_{2}<\xi_{3}(\bar{S})$. On peut conclure que l'équilibre $\bar{E}$ existe si $\lambda_{1}<\lambda_{2}<S^{0}, \xi_{1}\left(S^{0}\right)<\xi_{2}\left(S^{0}\right)$ et $\bar{X}_{2}<\xi_{3}(\bar{S})$ (voir Fig. 3).

\subsection{Stabilité locale}

On se propose de déterminer si les équilibres du système (1) sont LAS. La matrice Jacobienne en $\left(S, X_{1}, X_{2}\right)$ est :

$$
\mathbf{J}=\left(\begin{array}{ccc}
-m_{11} & -m_{12} & -m_{13} \\
m_{21} & m_{22} & -m_{23} \\
m_{31} & 0 & -m_{33}
\end{array}\right)
$$

avec

$$
m_{11}=D+\frac{f_{1}^{\prime}(S)}{y_{1}} X_{1}+\frac{f_{2}^{\prime}(S)}{y_{2}} X_{2}, \quad m_{12}=\frac{f_{1}(S)}{y_{1}}, \quad m_{13}=\frac{f_{2}(S)}{y_{2}}
$$




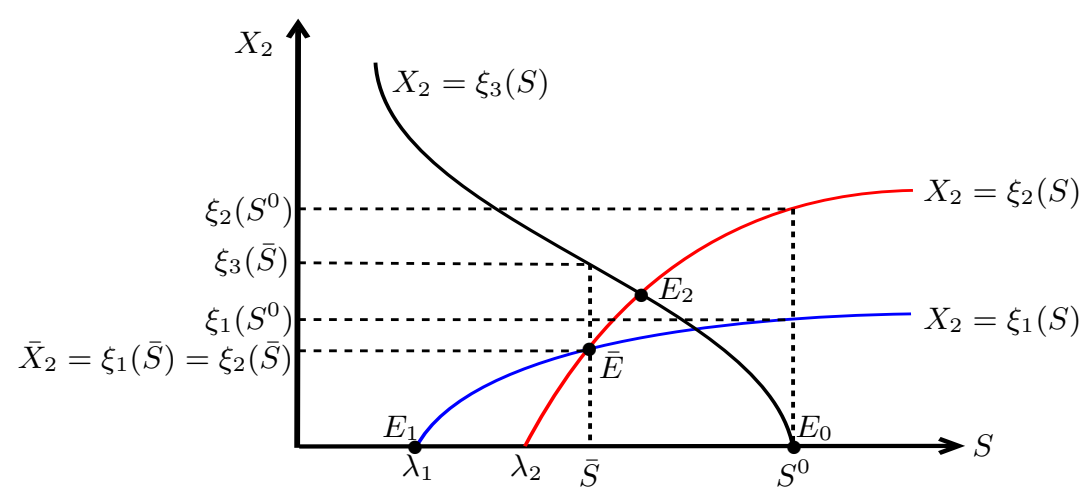

Figure 3. Condition d'existence du point d'équilibre $\bar{E}$.

$$
\begin{gathered}
m_{21}=f_{1}^{\prime}(S) X_{1}, \quad m_{22}=f_{1}(S)-q_{1}\left(X_{2}\right)-D_{1}, \quad m_{23}=q_{1}^{\prime}\left(X_{2}\right) X_{1}, \\
m_{31}=f_{2}^{\prime}(S) X_{2}, \quad m_{33}=q_{2}^{\prime}\left(X_{2}\right) X_{2}-\left[f_{2}(S)-q_{2}\left(X_{2}\right)-D_{2}\right] .
\end{gathered}
$$

Proposition $3.2 E_{0}$ est LAS si et seulement si $S^{0}<\lambda_{i}$ pour $i=1,2$.

Preuve. La matrice Jacobienne en $E_{0}$ est égale à

$$
\mathbf{J}_{\mathbf{0}}=\left(\begin{array}{ccc}
-D & -\frac{f_{1}\left(S^{0}\right)}{y_{1}} & -\frac{f_{2}\left(S^{0}\right)}{y_{2}} \\
0 & f_{1}\left(S^{0}\right)^{-}-D_{1} & 0 \\
0 & 0 & f_{2}\left(S^{0}\right)-D_{2}
\end{array}\right)
$$

Donc l'équilibre $E_{0}$ est LAS si et seulement si $f_{1}\left(S^{0}\right)<D_{1}$ et $f_{2}\left(S^{0}\right)<D_{2}$.

Remarquons que si $E_{0}$ est LAS, alors on ne peut pas avoir l'existence ni de $E_{1}$ ni de $E_{2}$ ni de $\bar{E}$.

Proposition 3.3 $E_{1}$ est LAS si et seulement si $\lambda_{1}<\lambda_{2}$ et $\lambda_{1}<S^{0}$.

Preuve. On sait que $E_{1}$ existe si et seulement si $\lambda_{1}<S^{0}$. La matrice Jacobienne du système (1) en $E_{1}$ est la même que celle du système (2). D'où le résultat.

Proposition 3.4 $E_{2}$ est LAS si et seulement si $\lambda_{2}<S^{0}$ et $f_{1}(\hat{S})-D_{1}<q_{1}\left(\hat{X}_{2}\right)$ c'est à dire $\xi_{1}(\hat{S})<\xi_{2}(\hat{S})=\xi_{3}(\hat{S})$.

Preuve. La matrice Jacobienne en $E_{2}$ est égale à

$$
\mathbf{J}_{\mathbf{2}}=\left(\begin{array}{ccc}
-D-\frac{f_{2}^{\prime}(\hat{S})}{y_{2}} \hat{X}_{2} & -\frac{f_{1}(\hat{S})}{y_{1}} & -\frac{f_{2}(\hat{S})}{y_{2}} \\
0 & f_{1}(\hat{S})-q_{1}\left(\hat{X}_{2}\right)-D_{1} & 0 \\
f_{2}^{\prime}(\hat{S}) \hat{X}_{2} & 0 & -q_{2}^{\prime}\left(\hat{X}_{2}\right) \hat{X}_{2}
\end{array}\right)
$$

Donc $f_{1}(\hat{S})-q_{1}\left(\hat{X}_{2}\right)-D_{1}$ est une valeur propre de $\mathbf{J}_{2}$. Cette valeur propre est négative si et seulement si $f_{1}(\hat{S})-D_{1}<q_{1}\left(\hat{X}_{2}\right)$. Les deux autres valeurs propres de $\mathbf{J}_{2}$ sont les valeurs propres de la matrice

$$
A=\left(\begin{array}{cc}
-D-\frac{f_{2}^{\prime}(\hat{S})}{y_{2}} \hat{X}_{2} & -\frac{f_{2}(\hat{S})}{y_{2}} \\
f_{2}^{\prime}(\hat{S}) \hat{X}_{2} & -q_{2}^{\prime}\left(\hat{X}_{2}\right) \hat{X}_{2}
\end{array}\right)
$$

Vol. 14 - pp. $15-30$ 
On a

$$
\begin{gathered}
\operatorname{det} A=q_{2}^{\prime}\left(\hat{X}_{2}\right) \hat{X}_{2}\left(D+\frac{f_{2}^{\prime}(\hat{S})}{y_{2}} \hat{X}_{2}\right)+\frac{f_{2}(\hat{S})}{y_{2}} f_{2}^{\prime}(\hat{S}) \hat{X}_{2}>0 \\
\operatorname{tr} A=-\left(D+\frac{f^{\prime}(\hat{S})}{y_{2}} \hat{X}_{2}+q_{2}^{\prime}\left(\hat{X}_{2}\right) \hat{X}_{2}\right)<0 .
\end{gathered}
$$

Par conséquent, les deux valeurs propres de $A$ sont à partie réelle strictement négative.

Proposition 3.5 $\bar{E}$ est LAS si et seulement si $\xi_{2}^{\prime}(\bar{S})<\xi_{1}^{\prime}(\bar{S})$.

Preuve. Supposons que l'équilibre $\bar{E}$ existe. La matrice Jacobienne en $\bar{E}$ est égale à

$$
\mathbf{J}_{\mathbf{E}}=\left(\begin{array}{ccc}
-m_{11} & -m_{12} & -m_{13} \\
m_{21} & 0 & -m_{23} \\
m_{31} & 0 & -m_{33}
\end{array}\right)
$$

avec

$$
\begin{gathered}
m_{11}=D+\frac{f_{1}^{\prime}(\bar{S})}{y_{1}} \bar{X}_{1}+\frac{f_{2}^{\prime}(\bar{S})}{y_{2}} \bar{X}_{2}, \quad m_{12}=\frac{f_{1}(\bar{S})}{y_{1}}, \quad m_{13}=\frac{f_{2}(\bar{S})}{y_{2}}, \\
m_{21}=f_{1}^{\prime}(\bar{S}) \bar{X}_{1}, \quad m_{23}=q_{1}^{\prime}\left(\bar{X}_{2}\right) \bar{X}_{1}, \quad m_{31}=f_{2}^{\prime}(\bar{S}) \bar{X}_{2}, \quad m_{33}=q_{2}^{\prime}\left(\bar{X}_{2}\right) \bar{X}_{2} .
\end{gathered}
$$

Le polynôme caractéristique est donné par

$$
P_{J}(\lambda)=a_{0} \lambda^{3}+a_{1} \lambda^{2}+a_{2} \lambda+a_{3}
$$

avec

$$
\begin{gathered}
a_{0}=-1, \quad a_{1}=-\left(m_{33}+m_{11}\right), \quad a_{2}=-\left(m_{33} m_{11}+m_{12} m_{21}+m_{31} m_{13}\right) \\
a_{3}=m_{12} m_{31} m_{23}-m_{12} m_{21} m_{33} .
\end{gathered}
$$

D'après le critère de Routh-Hurwitz, $\bar{E}$ est LAS si et seulement si on a

$$
\left\{\begin{array}{l}
a_{i}<0, \\
a_{1} a_{2}-a_{0} a_{3}>0 .
\end{array} \quad i=0 \cdots 3\right.
$$

Ce qui est équivalent à

$$
m_{31} m_{23}<m_{21} m_{33}
$$

c'est à dire $f_{2}^{\prime}(\bar{S}) \bar{X}_{2} q_{1}^{\prime}\left(\bar{X}_{2}\right) \bar{X}_{1}<f_{1}^{\prime}(\bar{S}) \bar{X}_{1} q_{2}^{\prime}\left(\bar{X}_{2}\right) \bar{X}_{2}$, soit $\xi_{2}^{\prime}(\bar{S})<\xi_{1}^{\prime}(\bar{S})$.

Sur la Fig. 4, on voit qu'il y a deux points d'équilibre $\bar{E}_{1}$ et $\bar{E}_{2}$, car l'équation $\xi_{1}(S)=$ $\xi_{2}(S)$ possède deux solutions $\bar{S}_{1}$ et $\bar{S}_{2}$. Le point d'équilibre $\bar{E}_{1}$ est instable puisque $\xi_{2}^{\prime}\left(\bar{S}_{1}\right)>\xi_{1}^{\prime}\left(\bar{S}_{1}\right)$, tandis que le point d'équilibre $\bar{E}_{2}$ est LAS car $\xi_{2}^{\prime}\left(\bar{S}_{2}\right)<\xi_{1}^{\prime}\left(\bar{S}_{2}\right)$.

L'ajout des termes de compétition interspécifique $q_{1}\left(X_{2}\right)$ et intraspécifique $q_{2}\left(X_{2}\right)$, a pu faire apparaître au moins un point d'équilibre positif localement asymptotiquement stable, c'est à dire que pour $\lambda_{1}<\lambda_{2}<S^{0}$ et selon la condition initiale, les deux espèces peuvent coexister.

Dans la suite, on se propose d'étudier la stabilité locale des points d'équilibre du système (1), selon le nombre $n$ de solutions de l'équation $\xi_{1}(S)=\xi_{2}(S)$ pour $0<S<\hat{S}$. On se place dans le cas générique où les intersections sont transverses et on note $\bar{E}_{i}$ les points d'équilibre positifs pour $i=1, \cdots, n$. 


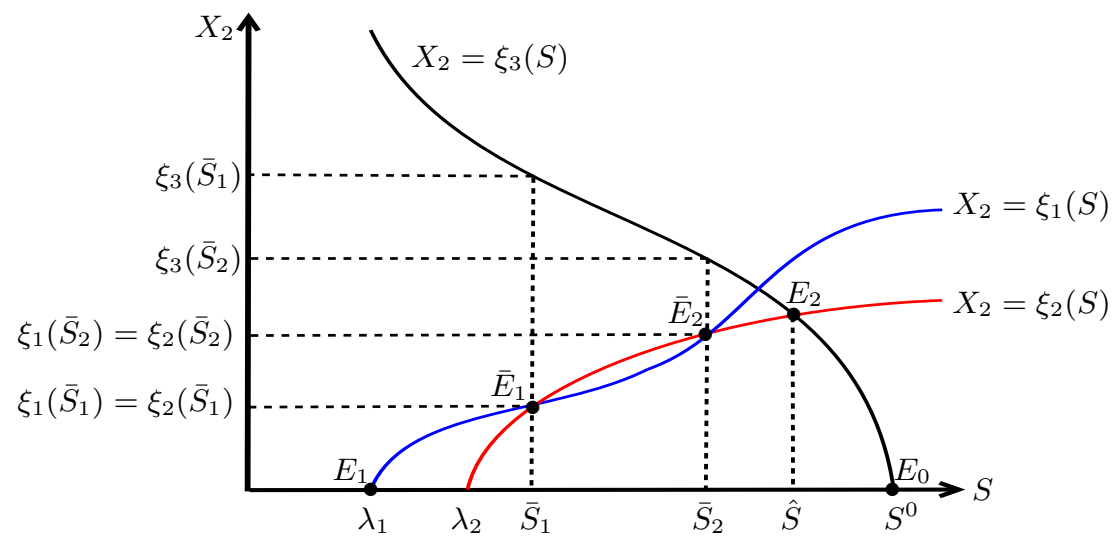

Figure 4. Stabilité locale de $\bar{E}_{2}$ et instabilité de $\bar{E}_{1}$.

Proposition 3.6 On suppose $\lambda_{1}<\lambda_{2}<S^{0}$, alors on a $E_{1}$ est stable et $E_{0}$ est instable. De plus,

- Si $n=0$, alors $E_{2}$ est instable

- Si $n=2 p$, alors $E_{2}$ est instable

- Si $n=2 p+1$, alors $\bar{E}_{2 p+1}$ est instable et $E_{2}$ est stable

avec $\bar{E}_{2 i-1}$ sont instables et $\bar{E}_{2 i}$ sont stables pour $1 \leqslant i \leqslant p$.

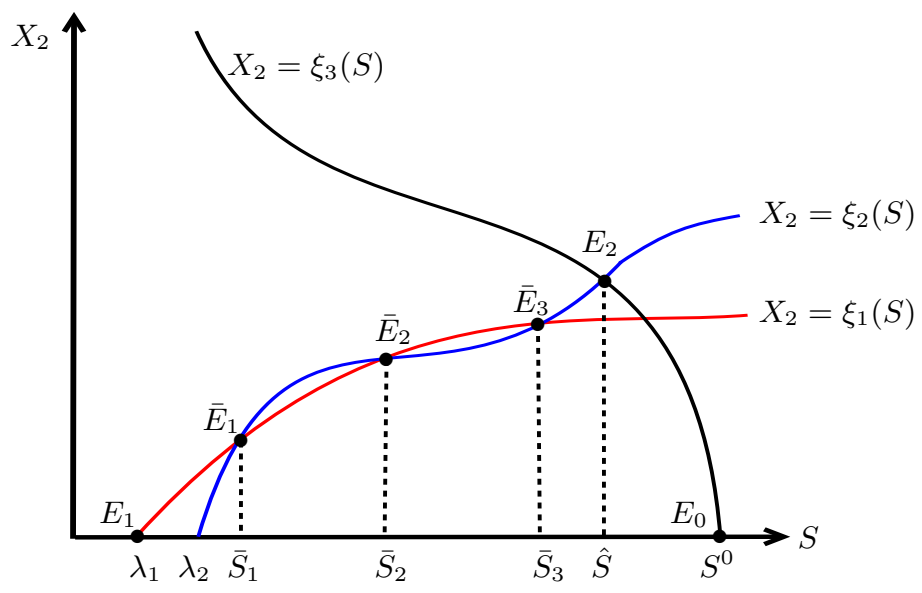

Figure 5. Cas $n=3$ : Stabilité locale de $E_{1}, \bar{E}_{2}, E_{2}$ et instabilité de $\bar{E}_{1}, \bar{E}_{3}, E_{0}$.

Preuve. On a toujours $E_{1}$ stable et $E_{0}$ instable, pour tout $\lambda_{1}<\lambda_{2}<S^{0}$. On suppose que l'équation $\xi_{1}(S)=\xi_{2}(S)$ admet $n=2 p+1$ solutions (voir Fig. 5)

$$
\begin{array}{ll}
\bar{S}_{2 i-1}, & i=1 \cdots p+1 \\
\bar{S}_{2 j}, & j=1 \cdots p .
\end{array}
$$

Comme les intersections sont transverses, alors

$$
\xi_{1}^{\prime}\left(\bar{S}_{2 i-1}\right)<\xi_{2}^{\prime}\left(\bar{S}_{2 i-1}\right) \quad \text { et } \quad \xi_{1}^{\prime}\left(\bar{S}_{2 j}\right)>\xi_{2}^{\prime}\left(\bar{S}_{2 j}\right)
$$

Vol. 14 - pp. $15-30$ 
D'après la Proposition 3.5, on déduit que $\bar{E}_{2 i-1}$ sont instables et $\bar{E}_{2 j}$ sont LAS.

Si $n=2 p+1$, alors $\xi_{1}(\hat{S})<\xi_{2}(\hat{S})$ et d'après la Proposition 3.4, on déduit que $E_{2}$ est LAS. Si $n=2 p$, alors $\xi_{1}(\hat{S})>\xi_{2}(\hat{S})$ et $E_{2}$ est instable. col.

Remarquons que si $\hat{S}$ est solution de l'équation $\xi_{1}(S)=\xi_{2}(S)$ alors $E_{2}$ est un noeud-

Le tableau suivant résume le résultat de la Proposition 3.6, où la lettre $\mathrm{S}$ signifie que l'équilibre est stable et la lettre I signifie que l'équilibre est instable.

\begin{tabular}{l||c|c|c|c|c|c|c|c} 
Équilibre & $E_{1}$ & $\bar{E}_{1}$ & $\bar{E}_{2}$ & $\cdots$ & $\bar{E}_{n-1}$ & $\bar{E}_{n}$ & $E_{2}$ & $E_{0}$ \\
\hline \hline$n$ impair & $\mathrm{S}$ & $\mathrm{I}$ & $\mathrm{S}$ & & $\mathrm{S}$ & $\mathrm{I}$ & $\mathrm{S}$ & $\mathrm{I}$ \\
\hline$n$ paire & $\mathrm{S}$ & $\mathrm{I}$ & $\mathrm{S}$ & & $\mathrm{I}$ & $\mathrm{S}$ & $\mathrm{I}$ & $\mathrm{I}$
\end{tabular}

\section{Simulations numériques}

On considère le modèle (1) où on prend le terme de compétition interspécifique linéaire égal à $d_{1} X_{2}$ ainsi que le terme de compétition intraspécifique linéaire égal à $d_{2} X_{2}$. On considère, comme taux de croissance, les fonctions croissantes $f_{i}(S)$ de type Monod, c'est à dire

$$
f_{i}(S)=\frac{m_{i} S}{a_{i}+S}, \quad i=1,2
$$

où $m_{i}$ désigne le taux de croissance maximal, $a_{i}$ est la constante de Monod et $d_{i}$ est le facteur inhibition de $X_{i} \operatorname{par} X_{2}$, pour $i=1,2$. Plus précisément, on se propose d'étudier le système

$$
\left\{\begin{array}{l}
\dot{S}=D\left(S^{0}-S\right)-\frac{1}{y_{1}} f_{1}(S) X_{1}-\frac{1}{y_{2}} f_{2}(S) X_{2} \\
\dot{X}_{1}=X_{1}\left(f_{1}(S)-d_{1} X_{2}-D_{1}\right) \\
\dot{X}_{2}=X_{2}\left(f_{2}(S)-d_{2} X_{2}-D_{2}\right) .
\end{array}\right.
$$

Proposition 4.1 Dans le modèle (5), l'équation $\xi_{1}(S)=\xi_{2}(S)$ admet au plus deux solutions.

Preuve. Comme les taux de croissance $f_{i}$ sont de type Monod et les termes de compétition $q_{i}$ sont linéaires, l'équation $\xi_{1}(S)=\xi_{2}(S)$ s'écrit

$$
\frac{\frac{m_{1}}{d_{1}} S}{a_{1}+S}-\frac{D_{1}}{d_{1}}=\frac{\frac{m_{2}}{d_{2}} S}{a_{2}+S}-\frac{D_{2}}{d_{2}}
$$

Cette équation équivaut à une équation du second degré en $S$ qui admet au plus deux racines.

Dans toutes les simulations, on fixe les paramètres suivants

$$
d_{1}=1, \quad d_{2}=1, \quad y_{1}=1, \quad y_{2}=1 \quad \text { et } \quad D=1 .
$$

Dans le premier cas, on choisit les paramètres

$$
m_{1}=2, \quad m_{2}=1.5, \quad a_{1}=2, \quad a_{2}=0.5, \quad D_{1}=0.5 \quad \text { et } \quad D_{2}=1
$$


de manière à ce que l'équation $\xi_{1}(S)=\xi_{2}(S)$ n'admet pas de solution (voir Fig. 6 à gauche), alors le point d'équilibre positif $\bar{E}$ n'existe pas. La solution de l'équation $\xi_{2}(S)=\xi_{3}(S)$, ainsi que les seuils de rentabilité, sont déterminés numériquement par les valeurs suivantes

$$
\hat{S}=1.796, \quad \lambda_{1}=0.666 \quad \text { et } \quad \lambda_{2}=1 .
$$

On considère la concentration du substrat à l'entrée du chémostat $S^{0}=2$. Les autres points d'équilibre sont donnés par

$$
E_{0}=(2,0,0), \quad E_{1}=(0.666,2.666,0) \quad \text { et } \quad E_{2}=(1.796,0,0.173) .
$$

Comme $\xi_{2}(\hat{S})<\xi_{1}(\hat{S})$, alors d'après la Proposition 3.4, le point d'équilibre $E_{2}$ est instable. De plus, on a $\lambda_{1}<\lambda_{2}<S^{0}$, alors le point d'équilibre $E_{1}$ est LAS et on a exclusion compétitive de la deuxième espèce par la première espèce (voir Fig. 6 à droite) pour les conditions initiales

$$
S(0)=2, \quad X_{1}(0)=0.5 \quad \text { et } \quad X_{2}(0)=1 .
$$
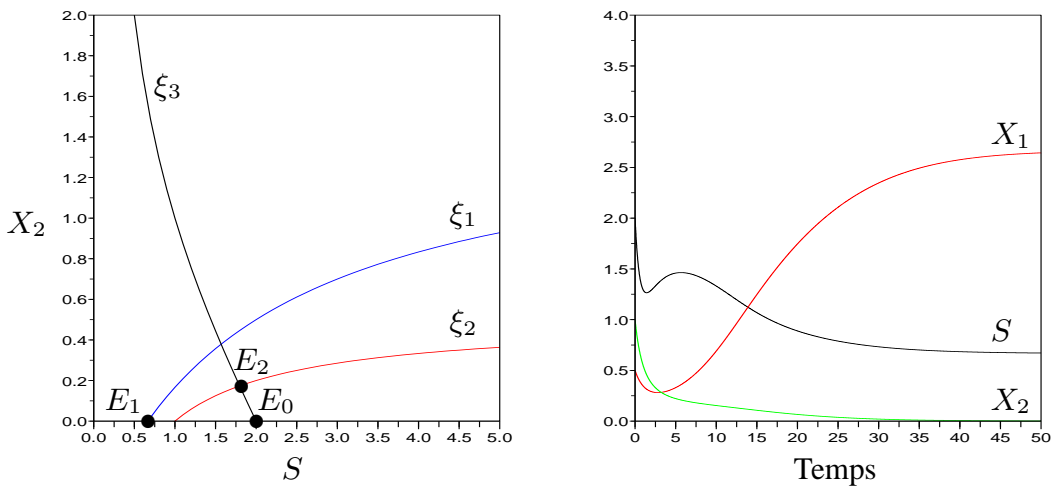

Figure 6. Exclusion compétitive de la deuxième espèce par l'espèce la plus compétitive.

Dans le deuxième cas, on modifie les paramètres

$$
m_{1}=1, \quad m_{2}=2, \quad a_{2}=2.5 \quad \text { et } \quad S^{0}=4
$$

tel qu'on a une seule solution de l'équation $\xi_{1}(S)=\xi_{2}(S)$ et la condition de stabilité locale de $E_{2}$ est vérifiée, c'est à dire $\xi_{1}(\hat{S})<\xi_{2}(\hat{S})$ (voir Fig. 7 à gauche).

Les valeurs numériques des seuils de rentabilité sont données par

$$
\lambda_{1}=2 \quad \text { et } \quad \lambda_{2}=2.5 \text {. }
$$

Les points d'équilibre sont donnés par

$E_{0}=(4,0,0), \quad E_{1}=(2,4,0), \quad E_{2}=(3.75,0,0.201) \quad$ et $\quad \bar{E}=(3.108,1.267,0.108)$.

La Fig. 7 à droite montre que la deuxième espèce a pu gagner la compétition sur la première espèce, pour les conditions initiales

$$
S(0)=1, \quad X_{1}(0)=2 \quad \text { et } \quad X_{2}(0)=0.5 .
$$



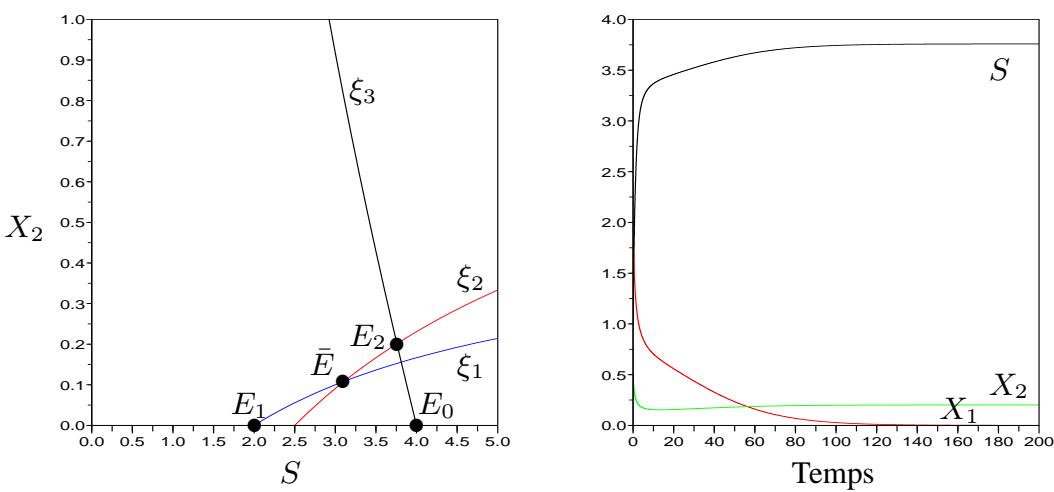

Figure 7. Exclusion compétitive de la première espèce par l'espèce la moins compétitive.

Dans ce cas, on a bistabilité avec deux bassins d'attraction où $E_{1}$ et $E_{2}$ sont LAS et $\bar{E}$ est instable.

Dans le troisième cas, on choisit les paramètres suivants

$$
m_{1}=2, \quad m_{2}=1, \quad a_{1}=2, \quad a_{2}=0.5, \quad D_{1}=0.1, \quad D_{2}=0.2 \quad \text { et } \quad S^{0}=1
$$

qui vérifient l'existence de deux solutions positives $\bar{S}_{1}$ et $\bar{S}_{2}$ de l'équation $\xi_{1}(S)=\xi_{2}(S)$, ainsi $\xi_{3}\left(\bar{S}_{2}\right)>\xi_{2}\left(\bar{S}_{2}\right)$, pour que l'équilibre positif $\bar{E}_{2}$ existe et soit LAS.

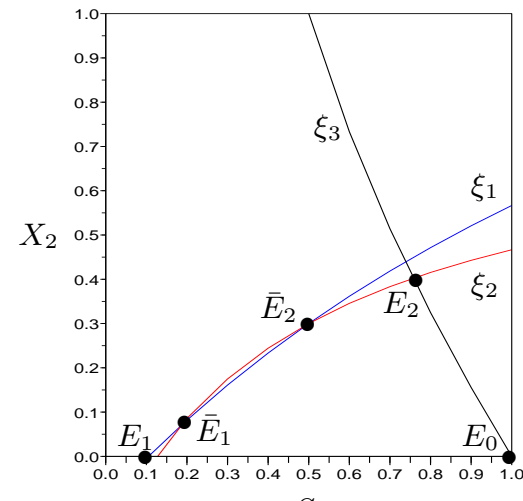

$S$

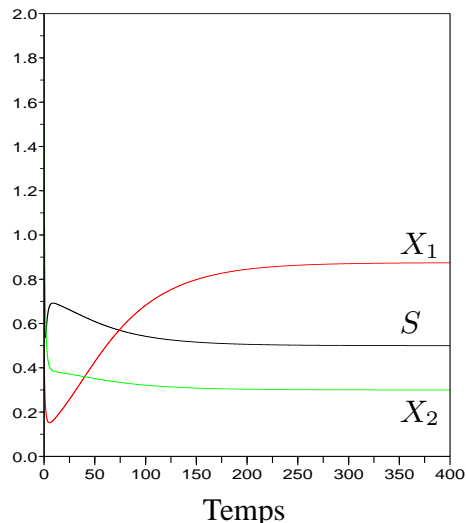

Temps

Figure 8. Bistabilité et coexistence

La Fig. 8 à gauche montre l'existence de deux points d'équilibre positifs et la figure à droite montre la coexistence entre les deux espèces pour les valeurs initiales suivantes

$$
S(0)=1, \quad X_{1}(0)=0.5 \quad \text { et } \quad X_{2}(0)=1.5 .
$$

Les valeurs numériques des seuils de rentabilité et les points d'équilibre stables sont

$$
\lambda_{1}=0.105, \quad \lambda_{2}=0.125, \quad E_{1}=(0.105,8.947,0) \quad \text { et } \quad \bar{E}_{2}=(0.5,0.87,0.3) .
$$

Les points d'équilibre instables sont

$$
\bar{E}_{1}=(0.181,4.802,0.066), \quad E_{2}=(0.757,0,0.402) \quad \text { et } \quad E_{0}=(1,0,0) .
$$




\section{Conclusion}

Dans ce travail, nous avons étudié le modèle mathématique de deux espèces microbiennes en compétition dans un chémostat pour une seule ressource. On a supposé que $\lambda_{1}<\lambda_{2}$ c'est à dire que la première espèce remporte la compétition sur la deuxième espèce pour des termes de compétition spécifiques nuls. Dans ce cas, le point d'équilibre $E_{1}$ correspondant à la concentration de la première espèce non nulle et la deuxième espèce nulle, est globalement asymptotiquement stable : c'est le Principe d'Exclusion Compétitive $[11,12,13]$. L'ajout du terme de compétition interspécifique $q_{1}\left(X_{2}\right)$ pour inhiber la croissance de $X_{1}$ par $X_{2}$, a pu faire apparaitre un équilibre positif $\bar{E}$, où les deux espèces coexistent, mais qui est instable. Ensuite, on a ajouté un terme de compétition intraspécifique $q_{2}\left(X_{2}\right)$ entre les individus de la deuxième espèce et on a montré que le point d'équilibre $E_{2}$, correspondant à la concentration de la première espèce nulle et la deuxième espèce non nulle, peut gagner de la stabilité locale. On a montré aussi l'existence d'un ou plusieurs points d'équilibre positifs localement asymptotiquement stables.

Noter que $E_{1}$ reste toujours LAS même lorsque les équilibres $E_{2}$ et $\bar{E}$ sont stables. Donc $E_{2}$ et $\bar{E}$ ne peuvent jamais être globalement asymptotiquement stables. Selon la condition initiale, la solution converge soit vers $E_{1}$ soit vers $E_{2}$ ou $\bar{E}$ selon le cas. Les simulations numériques illustrent les résultats mathématiques démontrés.

Remerciements : Les auteurs remercient les projets TREASURE (INRIA Euromed 3+3 project, http ://www.treasure.fr) et COADVISE (project FP7-PEOPLE-IRSES-2008 No. 230833) pour leur soutien.

\section{Bibliographie}

[1] P. De Leenheer, D. Angeli, E.D Sontag, « A feedback perspective for chemostat models with crowding effects », in Lecture Notes in Control and Inform Sci., Springer, vol. 294 (2003), 167-174.

[2] P. De Leenheer, D. Angeli, E.D Sontag, « Crowding effects promote coexistence in the chemostat », J. Math. Anal. Appl., vol. 319 (2006), 48-60.

[3] J. Hesseler, J.K. Schmidt, U. Reichl, D. Flockerzi, « Coexistence in the chemostat as a result of metabolic by-products », J. Math. Biol, vol. 53, n 4 (2006), 556-584.

[4] J.K. SCHMIdT, B. KÖNIG, U. REICHL, « Characterization of a Three Bacteria Mixed Culture in a Chemostat : Evaluation and Application of a Quantitative Terminal-Restriction Fragment Lenght Polymorphism (T-RFLP) Analysis for Absolute and Species Cell Enumeration », Biotechnology and Bioengineering, vol. 96, $\mathrm{n}^{\circ} 4$ (2007), 738-756.

[5] B. Haegeman, C. Lobry, J. Harmand, « Modelling Bacteria Flocculation as DensityDependent Growth », AIChE, vol. 53, n ${ }^{\circ} 2$ (2007), 535-539.

[6] B. HAegeman, A. RAPAPORT, « How flocculation can explain coexistence in the chemostat », J. Biol. Dyn., vol. 2, $\mathrm{n}^{\circ} 1$ (2008), 1-13.

[7] C. LOBRY, J. HARMAND, «A new hypothesis to explain the coexistence of $n$ species in the presence of a single resource », C. R. Biologies, vol. 329 (2006), 40-46.

[8] C. LobRy, F. MAZENC, « Effect on persistence of intra-specific competition in competition models », Electron. J. Differential Equations, $\mathrm{n}^{\circ} 125$ (2007), 1-10.

Vol. 14 - pp. $15-30$ 
30 A R I M A - Volume 14-2011

[9] C. Lobry, F. MAZEnC, A. RAPAPORT, «Persistence in ecological models of competition for a single ressource », C. R. Acad. Sci. Paris, Ser. I, vol. 340 (2005), 199-204.

[10] A. RAPAPORT, J. HARMAND, «Biological control of the chemostat with nonmonotone response and different removal rates », Math. Biosci. Eng., vol. 5, n 3 (2008), 539-547.

[11] T. SARI, «A Lyapunov function for the chemostat with variable yields », C. R. Math. Acad. Sci. Paris, vol. 348, 13-14 (2010), 747-751.

[12] T. SARI, F. MAZENC, « Global dynamics of the chemostat with different removal rates and variable yields », Math. Biosci. Eng.vol. 8, n ${ }^{\circ} 3$ (2011), 827-840.

[13] H.L. Smith, P. Waltman, « The Theory of the Chemostat, Dynamics of Microbial Competition », Cambridge University Press, 1995.

[14] G.S.K. Wolkowicz, L. ZHIQI, « Direct interference on competition in a chemostat », J. Biomath, vol. 13, n 3 (1998), 282-291. 